\title{
NudC regulates actin dynamics and ciliogenesis by stabilizing cofilin 1
}

Cheng Zhang, ${ }^{1, *}$, Wen Zhang ${ }^{1, *}$, Yi Lu ${ }^{1, *}$, Xiaoyi Yan ${ }^{1,2}$, Xiumin Yan $^{3}$, Xueliang Zhu ${ }^{3}$, Wei Liu ${ }^{1}$, Yuehong Yang ${ }^{1,2}$, Tianhua Zhou ${ }^{1,2}$

${ }^{l}$ Department of Cell Biology and Program in Molecular Cell Biology, Zhejiang University School of Medicine, Hangzhou, Zhejiang 310058, China, ${ }^{2}$ Collaborative Innovation Center for Diagnosis and Treatment of Infectious Diseases, Hangzhou, Zhejiang 310003, China, ${ }^{3}$ State Key Laboratory of Cell Biology, Institute of Biochemistry and Cell Biology, Shanghai Institutes for Biological Sciences, Chinese Academy of Sciences, 320 Yue Yang Road, Shanghai 200031, China

Emerging data indicate that actin dynamics is associated with ciliogenesis. However, the underlying mechanism remains unclear. Here we find that nuclear distribution gene $\mathrm{C}(\mathrm{NudC})$, an $\mathrm{Hsp90}$ co-chaperone, is required for actin organization and dynamics. Depletion of NudC promotes cilia elongation and increases the percentage of ciliated cells. Further results show that NudC binds to and stabilizes cofilin 1, a key regulator of actin dynamics. Knockdown of cofilin 1 also facilitates ciliogenesis. Moreover, depletion of either NudC or cofilin 1 causes similar ciliary defects in zebrafish, including curved body, pericardial edema and defective left-right asymmetry. Ectopic expression of cofilin 1 significantly reverses the phenotypes induced by NudC depletion in both cultured cells and zebrafish. Thus, our data suggest that NudC regulates actin cytoskeleton and ciliogenesis by stabilizing cofilin 1 .

Keywords: NudC; actin dynamics; ciliogenesis; cofilin 1; zebrafish development

Cell Research (2016) 26:239-253. doi:10.1038/cr.2015.152; published online 25 December 2015

\section{Introduction}

Cilia are evolutionarily conserved microtubule-based organelles that project from the cell surface. In vertebrates, cilia are classified as motile or nonmotile (primary). Motile cilia often occur in epithelial tissues to generate fluid flow, while non-motile cilia are distributed widely and are considered critical for extracellular signal reception and transduction $[1,2]$. Cilia play essential roles in vertebrate development, including establishment of left-right asymmetry, and brain and kidney development [3-5]. Disruption of cilia structure or motility is associated with a range of human disorders termed ciliop-

\footnotetext{
*These three authors contributed equally to this work. Correspondence: Tianhua Zhou ${ }^{\mathrm{a}}$, Yuehong Yang ${ }^{\mathrm{b}}$

${ }^{a}$ Tel: +8657188208258

E-mail: tzhou@zju.edu.cn

${ }^{\mathrm{b}}$ Tel: +86571 88208257

E-mail: yhyang@zju.edu.cn

Received 22 April 2015; revised 22 September 2015; accepted 15 October 2015; published online 25 December 2015
}

athies, such as primary ciliary dyskinesias, cystic kidney diseases and situs inversus [6-9].

Accumulating data suggest that actin dynamics affects ciliogenesis. Either silencing of actin-related protein ARP3 or treatment with actin polymerization inhibitor cytochalasin D facilitates cilia formation and promotes cilia elongation in mammalian cells [10]. Recently, we found that miR-129-3p, a microRNA conserved in vertebrates, promotes both ciliogenesis and the axoneme growth by repressing branched F-actin formation [11]. However, the regulation of ciliogenesis by actin dynamics remains elusive.

Nuclear distribution gene $\mathrm{C}$ (NudC) was first identified as a regulator of nuclear movement in the filamentous fungus Aspergillus nidulans [12]. We and other groups have found that NudC plays crucial roles in diverse cellular processes, including cell division and neuronal migration, partially by modulating the dynein complex [13-15]. A growing body of evidence implies that NudC functions as a co-chaperone of Hsp90 to stabilize client proteins [16-18]. Proteomics data from our laboratory and others indicate that NudC may interact with cofilin 
$1[17,18]$, a key regulator of actin dynamics, yet little is known about the role of NudC in actin cytoskeleton.

In this report, we find that NudC is essential for actin organization and dynamics by regulating the stability of cofilin 1. Depletion of either NudC or cofilin 1 facilitates ciliogenesis in mammalian cells and results in ciliary defects in zebrafish. More importantly, exogenous expression of cofilin 1 significantly reverses the phenotypes caused by NudC knockdown in either cultured cells or zebrafish. These results indicate a critical role of NudC in actin cytoskeleton and ciliogenesis by stabilizing cofilin 1 .

\section{Results}

\section{Depletion of NudC influences actin dynamics}

To determine the role of NudC in actin cytoskeleton, we first examined the cellular localization of NudC together with actin in RPE-1 cells. The data revealed that NudC was mainly localized in cytoplasm and concentrated in lamellipodia (Supplementary information, Figure S1). Then, we employed lentivirus-mediated RNAi targeting two different regions of $N u d C$ mRNA in RPE1 cells and found that the protein level of NudC was substantially reduced (Figure 1A). Immunostaining results showed that thick stress fibers were frequently detected in RPE-1 cells depleted of NudC (Figure 1B), which was reversed by ectopic expression of RNAi-resistant NudC (Supplementary information, Figure S2). Scratch wound assays further displayed that NudC depletion significantly inhibited collective cell migration and lamellipodia formation at the leading edge (Figure 1C, 1D and Supplementary information, Figure S3). Kymographs of lamellipodial protrusion confirmed that knockdown of NudC led to decreases in protrusion velocity with concomitant increases in protrusion persistence (Figure 1E$1 \mathrm{G})$. Moreover, we also observed that NudC depletion resulted in impaired cell spreading (Figure 1H and 1I). These data strongly indicate that NudC is a crucial regulator of actin dynamics.

\section{NudC suppresses primary cilia assembly}

As it has been reported that actin dynamics affects ciliogenesis in mammalian cells $[10,11]$, we tested the potential role of NudC in primary cilia assembly. Given that cilia typically form during G1 or G0 phase and disassemble around the time of mitosis, serum starvation induces ciliogenesis and addition of serum inhibits ciliation $[6,19,20]$. In this study, cilia length was measured under serum deprivation and the percentage of ciliated cells was calculated under serum-rich condition. NudC depletion in RPE-1 cells caused a significant elongation of primary cilia in the absence of serum and increased the percentage of the ciliated cells in the presence of serum (Figure 2A-2F). Induction of ciliogenesis by NudC knockdown was significantly reversed by ectopic expression of RNAi-resistant NudC (Supplementary information, Figure S4). In consistence with the results above, knockdown of NudC in HEK293 T cells also substantially induced primary cilia assembly (Supplementary information, Figure S5). Given that ciliation is associated with cell cycle regulation [1, 21, 22], we determined whether NudC depletion influences cell cycle. Flow cytometry showed that there was no significant difference between the control and NudC-depleted RPE1 cells during cell cycle progression under our conditions (Figure $2 \mathrm{G}$ and $2 \mathrm{H}$ ). As inhibition of actin dynamics has been shown to induce ciliogenesis by accumulating ciliogenic membrane vesicles positive for Rab11a around the centrosome regions $[10,11,20]$, we tested whether NudC depletion promotes ciliogenesis via the similar mechanism. In NudC-depleted RPE-1 cells, Rab11a-positive vesicles were more frequently accumulated around the centrosome region compared with the control cells (Figure 2I and 2J). Furthermore, overexpression of NudC considerably inhibited ciliation in RPE-1 cells (Figure $2 \mathrm{~K}-2 \mathrm{~N}$ ). Thus, these results suggest that NudC plays an inhibitory role in ciliogenesis in mammalian cells.

NudC knockdown results in ciliary phenotypes in zebrafish

Cilia have been demonstrated to be involved in various developmental processes in vertebrates $[5,11,23$, 24]; therefore, we explored the function of NudC during zebrafish development. Zebrafish NudC shares high homology with human NudC (identity, $72.0 \%$; similarity, $85.0 \%)$ and the polyclonal antibodies against human NudC also recognized zebrafish NudC (Figure 3A). RTPCR analysis revealed that NudC mRNA was ubiquitously expressed in embryonic and early larval stages of zebrafish (Supplementary information, Figure S6A). Whole mount in situ hybridization showed that NudC was detected in the brain, eyes, pronephros and so on (Supplementary information, Figure S6B-S6I). We then designed antisense morpholino oligonucleotides (MOs) to block the translation of NudC mRNA (NudC MO-1) and found that injection with low dose of NudC MO-1 (2.5 ng per embryo) obviously decreased endogenous NudC in zebrafish embryos (Figure 3A). NudC morphants exhibited several ciliary defects such as curved body $(56.7 \%)$, pericardial edema $(87.7 \%)$ and hydrocephalus $(27.1 \%)$ at $72 \mathrm{~h}$ post fertilization (hpf) (Figure 3B and 3C). Histological cross-sections confirmed the existence of hydrocephalus and displayed dilation of anterior pronephric duct in NudC morphants (Figure 3D and 3E). Furthermore, we tested the position of the heart tube 
A

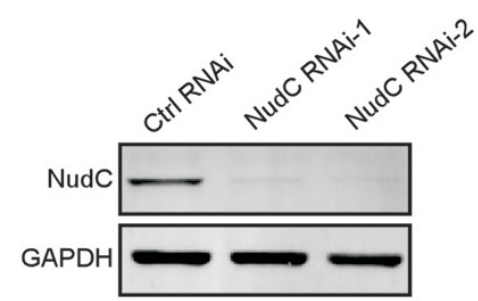

B

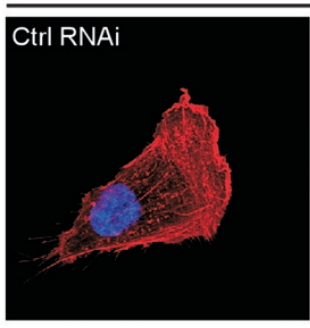

Phalloidin/DNA
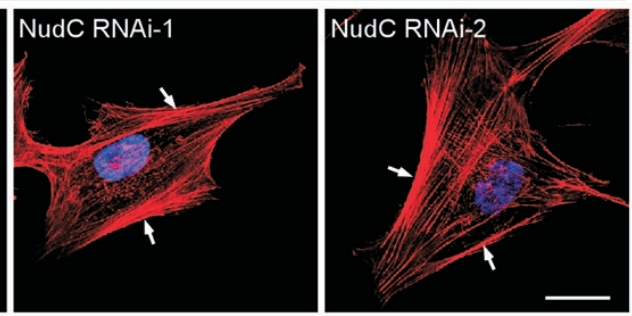

C

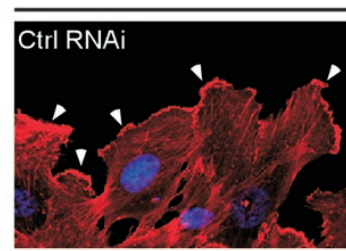

Phalloidin/DNA

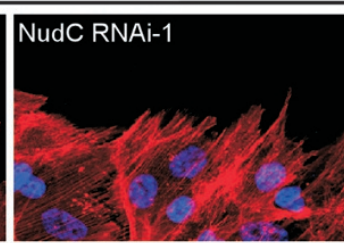

E

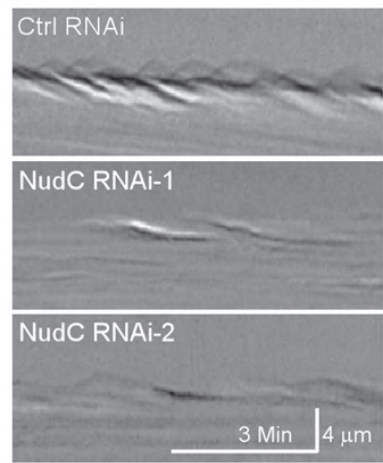

H

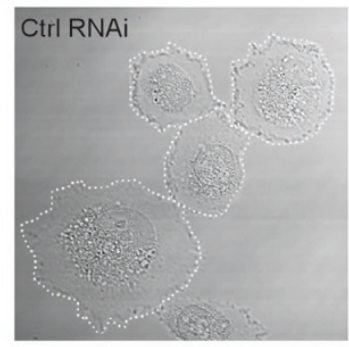

F
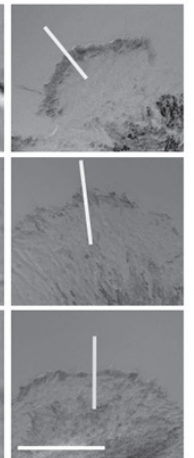

NudC RNAi-1
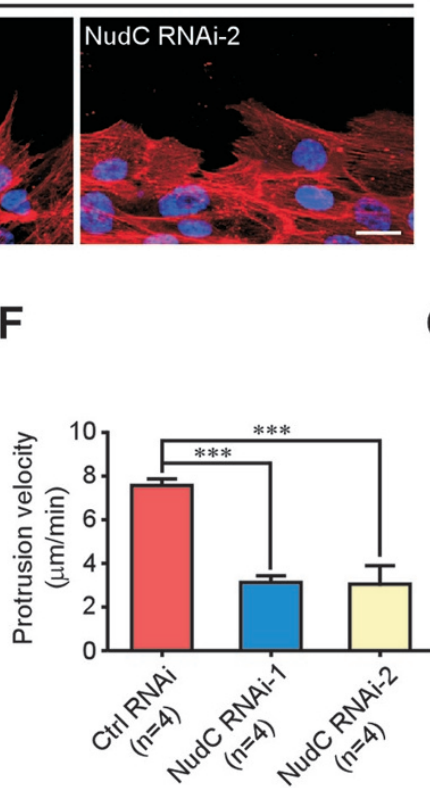

I
D

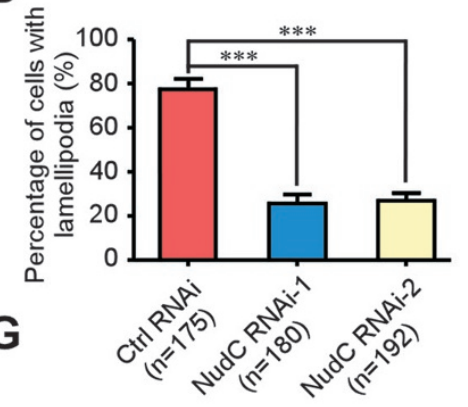

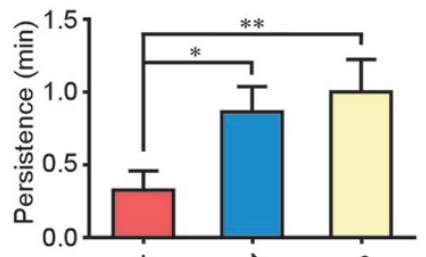
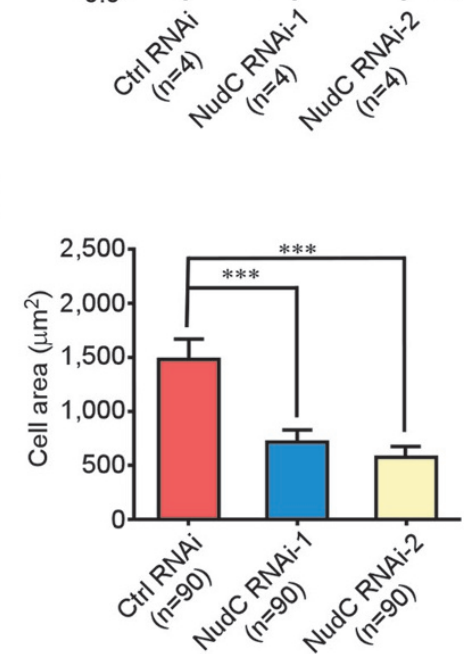

Figure 1 Depletion of NudC disrupts actin organization. RPE-1 cells were treated with lentivirus-delivered shRNAs, NudC RNAi-1 or -2 , targeting different regions of NudC. (A) Western blotting showing efficient suppression of NudC. GAPDH is the loading control. (B) Immunofluorescence revealing thick stress fibers (arrows) in NudC-depleted cells. (C, D) Immunostaining showing less lamellipodia formation (arrowheads) in cells depleted of NudC at the leading edge after scratching (C). Cells with lamellipodia are counted (D). More than 50 cells are analyzed per experiment. (E-G) Kymographs revealing decreases in protrusion dynamics of NudC-depleted cells. Minimum intensity projection of a 250 -frame movie ( $3 \mathrm{~s}$ per frame) is presented on the right. Pixel intensities along a one-pixel-wide line (white) are used to generate the kymograph presented on the left (E). The velocity $(\mathbf{F})$ and persistence $(\mathbf{G})$ of lamellipodia protrusion are calculated. (H, I) Phase contrast images showing impaired cell spreading in NudC knockdown cells. The areas of cell spreading are outlined by dashed lines and measured by Image $\mathrm{J}$ software (NIH). More than 20 cells are counted in each experiment. DNA is labeled with DAPI. Scale bars represent $20 \mu \mathrm{m}$. Quantitative data are derived from at least three independent experiments and expressed as mean \pm SD. $\mathrm{n}$, sample size. ${ }^{*} P$ $<0.05$; ** $P<0.01$; ***P 0.001 . 
A

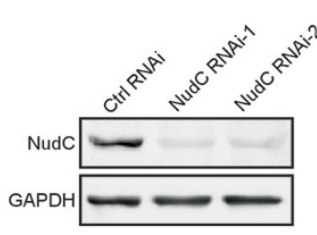

B

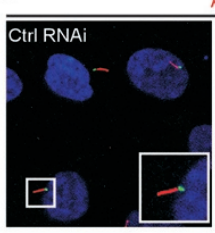

Arl13b/y-tub/DNA - serum

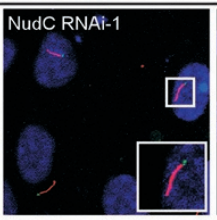

$\square$

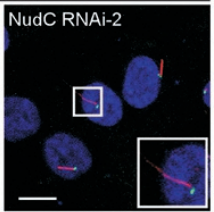

C

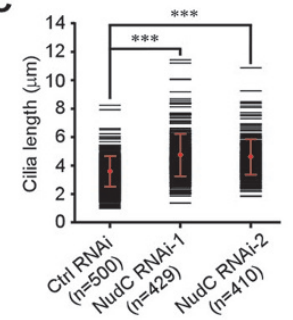

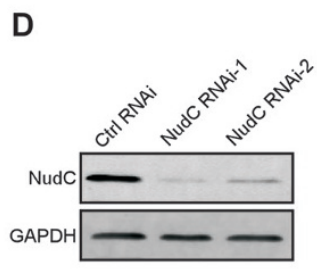

E
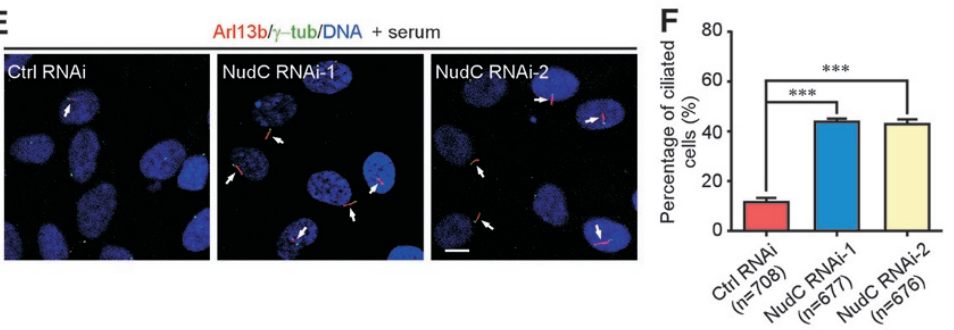

G
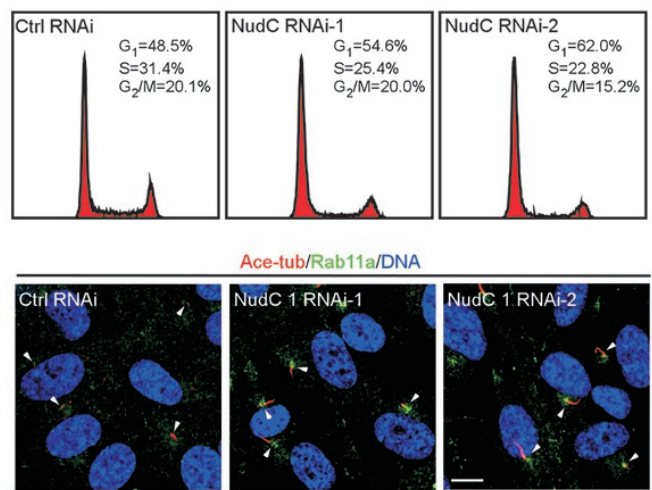

H

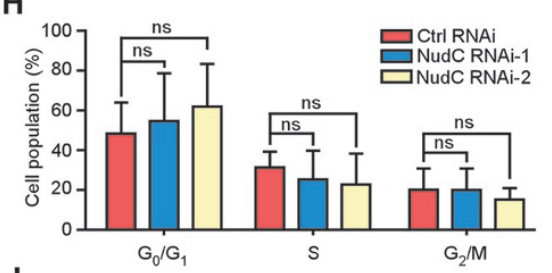

$\mathbf{J}$

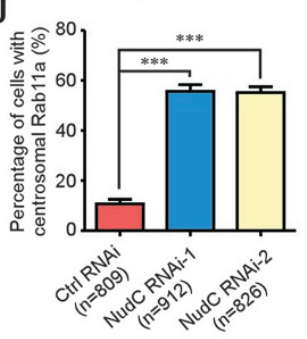

$$
\text { M }
$$

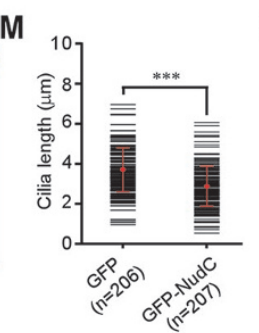

N

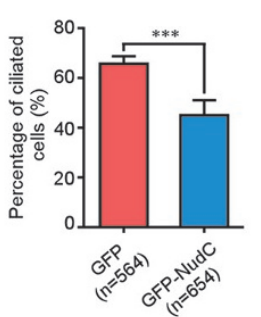

Figure 2 NudC suppresses ciliogenesis in mammalian cells. (A-C) RPE-1 cells treated with either NudC RNAi-1 or -2 under serum starvation are subjected to the following analyses. Immunoblotting confirms significant knockdown of NudC (A). GAPDH is the internal control. Immunofluorescence with anti-Arl13b (cilia marker) and $\gamma$-tubulin ( $\gamma$-tub) antibodies reveals an increase in cilia length after NudC depletion (B). Boxed regions are enlarged in insets. Lengths of more than 120 cilia (per experiment) are measured by Image $\mathrm{J}$ software (C). (D-J) RPE-1 cells treated with either NudC RNAi-1 or -2 are analyzed. Western blotting shows significant suppression of NudC (D). Immunostaining reveals spontaneous ciliogenesis (arrow) in NudC knockdown cells (E). Percentage of cells with cilia in each experiment (F). Flow cytometry reveals no significant difference between control and NudC-depleted cells during cell cycle progression (G, H). Immunofluorescence with anti-acetylated tubulin (ace-tub, cilia marker) and Rab11a antibodies reveals an increase in Rab11a-positive vesicles around the centrosome regions (arrowheads) in NudC-depleted cells (I, J). More than 150 cells are scored each time. (K-N) Overexpression of NudC suppresses ciliogenesis. RPE-1 cells transfected with the indicated vectors are subjected to western blotting (K) and immunostaining (L). Lengths of more than 60 cilia (per experiment) are measured by Image J software (M). Over 180 cells with cilia are counted in each experiment (N). DNA is visualized by DAPI. Scale bars represent $10 \mu \mathrm{m}$. Quantitative data from at least three independent experiments are shown as mean $\pm \mathrm{SD}$. $\mathrm{n}$, sample size. ${ }^{* * *} P<0.001$; ns, not significant $(P>0.05)$. 
(using cardiac myosin light chain 2, cmlc2, as a marker) and distribution of an early asymmetry-determining factor (lefty2) in NudC morphants. The results revealed that cmlc2 (34.8\%) and lefty2 (43.3\%) were not restricted to the left side of the embryos injected with $\mathrm{NudC}$ MO-1 (Figure 3F-3I), implying an essential role of NudC in controlling left-right asymmetry. Importantly, all of these defects caused by $N u d C$ MO-1 were effectively reversed by exogenous expression of $N u d C$ mRNA (Figure 3B, $3 \mathrm{C}, 3 \mathrm{G}$ and $3 \mathrm{I}$ ).

Given that Kupffer's vesicle (KV) is a ciliated organ that governs left-right asymmetry of zebrafish embryo $[25,26]$, we performed whole mount immunofluorescence (IF) and observed that NudC knockdown significantly increased cilia length in $\mathrm{KV}$, which was reversed by ectopic expression of $N u d C$ mRNA (Figure $3 \mathrm{~J}$ and $3 \mathrm{~K})$. As it has been reported that the motile cilia in pronephric ducts are crucial for fluid flow [5, 11, 23], we examined whether NudC influences the cilia in pronephric ducts. Immunostaining showed that NudC depletion caused longer cilia in anterior pronephric ducts of NudC morphants, which was reversed by exogenous expression of NudC mRNA (Figure 3L). Video microscopy showed that the cilia in enlarged pronephric ducts of NudC morphants were randomly orientated and beat in an uncoordinated manner, which were also significantly rescued by ectopic expression of $N u d C$ mRNA (Figure $3 \mathrm{M}$ and $3 \mathrm{~N}$; Supplementary information, Movies S1-S4).

In order to minimize the off-target effects of NudC MO, we employed another MO targeting a different region of NudC mRNA (NudC MO-2), and observed similar phenotypes to that of NudC MO-1, which were also significantly reversed by $N u d C$ mRNA (Supplementary information, Figure S7). A recent study showed that generation of robust biallelic mutations by CRISPR/ Cas9 system in the injected zebrafish embryos appears to allow for phenotypic analysis directly in the F0 animals [27]. To further confirm the function of NudC during zebrafish development, we designed a guide RNA (gRNA) to target zebrafish $N u d C$. Phenotypes of Cas9/NudC-gRNA-injected embryos were similar to that of NudC morphants (Supplementary information, Figure S8). These data together suggest a crucial role of NudC in ciliation and cilia-mediated developmental processes in zebrafish.

\section{NudC binds to and stabilizes cofilin 1}

Mass spectrometry data of proteins co-immunoprecipitated with NudC reported by our group and others have indicated that cofilin 1 may interact with NudC $[17,18]$. The similar roles of NudC and cofilin 1 in actin dynamics prompted us to investigate the biochemical interaction between these two proteins [28-30]. GST pull- down and immunoprecipitation experiments revealed that NudC associated with cofilin 1 (Figure 4A and 4B). More importantly, endogenous NudC was also able to interact with cofilin 1 in RPE-1 cells (Figure 4C and 4D). IF combined with scratch wound assay confirmed that NudC was colocalized with cofilin 1 at the leading edge (Figure 4E). These results strongly suggest that NudC forms a complex with cofilin 1 in mammalian cells.

Previous data from our laboratory and others suggest that NudC may be involved in the regulation of protein stability $[17,18]$. We tested whether NudC regulates the stability of cofilin 1 and found that NudC depletion substantially decreased the protein level of cofilin 1, but not its mRNA level (Figure 4F and 4G). Ubiquitin proteasome pathway is responsible for the targeted degradation of intracellular proteins such as cofilin $1[31,32]$; we thus investigated whether the proteasome pathway is involved in the regulation of cofilin 1 stability by NudC. Indeed, degradation of cofilin 1 after NudC depletion was blocked by MG132, a proteasome inhibitor (Figure $4 \mathrm{H}$ ), and NudC depletion significantly increased the ubiquitination of cofilin 1 (Figure 4I). Thus, NudC depletion may induce the degradation of cofilin 1 via the ubiquitin proteasome pathway.

\section{NudC acts upstream of cofilin 1 to regulate cilia assembly}

Based on the data that NudC influences ciliogenesis and cofilin 1 stability (Figures 2-4), we assumed that NudC may modulate ciliogenesis by regulating cofilin 1 stability. Depletion of cofilin 1 by two different small interfering RNAs (siRNAs) in RPE-1 cells caused a significant elongation of primary cilia in the absence of serum and increased the percentage of the ciliated cells in the presence of serum (Figure 5A-5F). Consistent with the data above, knockdown of cofilin 1 in HEK293T cells also considerably promoted cilia assembly (Supplementary information, Figure S9). More Rab11a-positive vesicles were observed around the centrosome regions in cofilin 1-depleted cells compared with the control (Figure $5 \mathrm{H}$ and $5 \mathrm{G}$ ). Furthermore, overexpression of cofilin 1 effectively inhibited ciliogenesis (Figure 5I-5L). These results indicate that cofilin 1 plays a similar role in ciliogenesis as NudC.

To determine whether NudC regulates ciliogenesis via modulating cofilin 1 expression, we performed RNAi rescue assays in mammalian cells. Exogenous expression of cofilin 1 significantly reversed the abnormal ciliogenesis induced by NudC depletion, but not vice versa (Figure $6 \mathrm{~A}-6 \mathrm{D}$ and $6 \mathrm{G}-6 \mathrm{~J})$. Furthermore, double knockdown of NudC and cofilin 1 did not show synergistic effects on ciliogenesis compared with that of cofilin 1 depletion (Figure $6 \mathrm{E}, 6 \mathrm{~F}, 6 \mathrm{~K}$ and $6 \mathrm{~L}$ ), suggesting that NudC and 


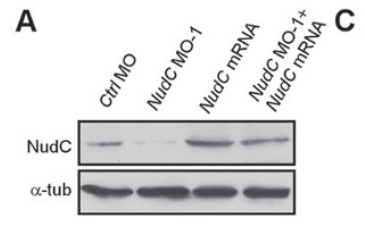

B
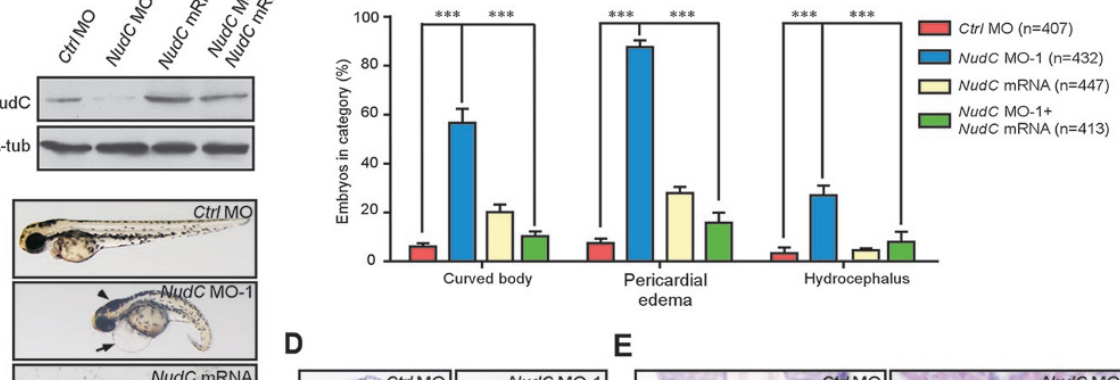

D

E
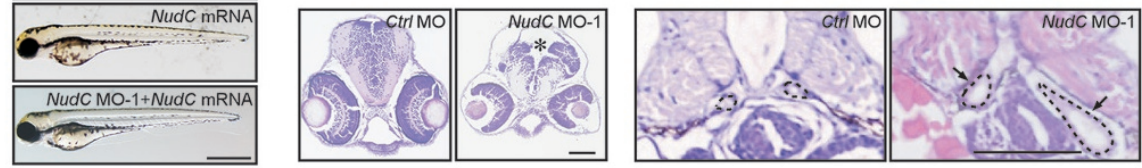

F
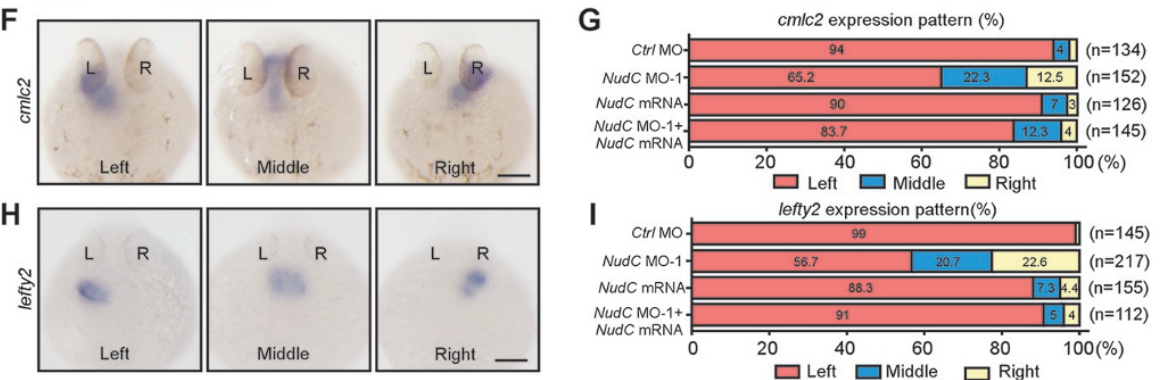
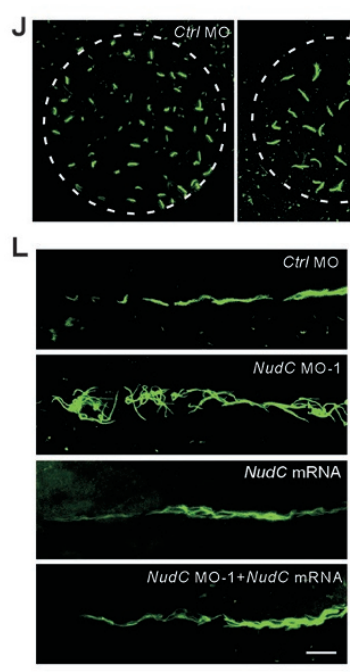

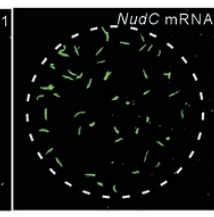

M

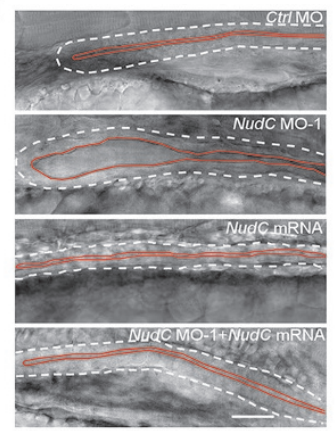

N
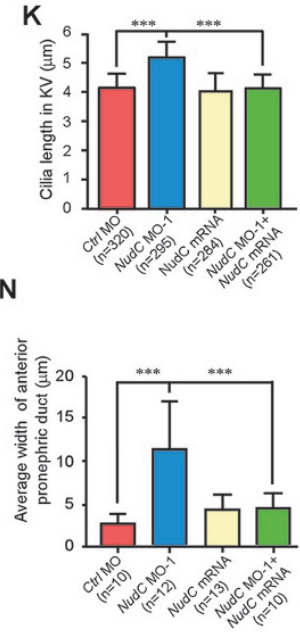

Figure 3 NudC depletion induces multiple ciliary defects in zebrafish. Embryos were injected with 2.5 ng control morpholino (Ctrl MO), $2.5 \mathrm{ng}$ NudC MO-1, 25 pg NudC mRNA or $2.5 \mathrm{ng}$ NudC MO-1 plus $25 \mathrm{pg}$ NudC mRNA and collected at the indicated times. (A) Western analysis showing efficiency of NudC knockdown. $\alpha$-tubulin ( $\alpha$-tub) is used as the internal control. (B, C) Bright-field micrographs of embryos showing body curvature, pericardial edema (arrow) and hydrocephalus (arrowhead) in NudC morphants at 72 hpf. Scale bar, $500 \mu \mathrm{m}$. (D, E) Sections of brain (D) and pronephric duct (E) of NudC morphants displaying hydrocephalus (asterisk) and dilated pronephric ducts (arrows) at $72 \mathrm{hpf}$. Black dashed lines indicate the boundaries of pronephric ducts. Scale bar, $200 \mu \mathrm{m}$. (F-I) Whole mount in situ hybridization with cmlc2 at $30 \mathrm{hpf}(\mathbf{F})$ or lefty2 at $22 \mathrm{hpf}(\mathbf{H})$ shows defective left-right asymmetry (dorsal view). Scale bar, $500 \mu \mathrm{m}$. Percentages of different expression patterns of $\mathrm{cm} / \mathrm{c} 2$ (G) or lefty2 (I) are also presented. (J, K) Immunofluorescence with anti-ace-tub antibody (green) reveals an increase in cilia length in KV of NudC morphants at 8-somite stage (8 ss). Scale bar, $10 \mu \mathrm{m}$. Cilia length in KV is measured using Image $\mathrm{J}$ software (K). (L) Immunostaining of anterior pronephric ducts shows longer cilia in NudC morphant at $24 \mathrm{hpf}$. Scale bar, 10 $\mu \mathrm{m}$. (M, N) Images extracted from video of anterior pronephric duct showing an enlarged lumen in NudC morphant at $48 \mathrm{hpf}$. Embryos (lateral views) are oriented with anterior to the left and dorsal up (M). White dashed lines and red lines indicate the boundaries and lumens of pronephric ducts, respectively. Scale bar, $20 \mu \mathrm{m}$. The width of lumen is measured using Image $\mathrm{J}$ software $(\mathbf{N})$. Data from at least three independent experiments are represented as mean $\pm \mathrm{SD}$. $\mathrm{n}$, sample size. $* * * P<0.001$; ns, not significant $(P>0.05)$. 
cofilin 1 may act in the same pathway to modulate ciliogenesis.

Cofilin 1 rescues the ciliary phenotypes in NudC morphants

To further investigate the role of cofilin 1 in ciliogenesis during zebrafish development, we used MO to deplete cofilin 1 and found that injection with low dose of cofilin 1 MO-1 (2.0 ng per embryo) effectively decreased endogenous cofilin 1 in embryos (Supplementary information, Figure S10A). Cofilin 1 morphants displayed several ciliary defects, including curved body $(42.0 \%)$ and pericardial edema ( $81.9 \%$; Figure $7 \mathrm{~A}$ and $7 \mathrm{~B})$, which were significantly reversed by exogenous expression of cofilin 1 mRNA (Supplementary information, Figure S10). Whole mount in situ hybridization revealed that cmlc2 was not restricted to the left side in $48.4 \%$ of embryos injected with cofilin $1 \mathrm{MO}-1$, and lefty 2 was not restricted to the left side in $46.3 \%$ of cofilin 1 morphants (Figure 7C-7F). Immunostaining of cofilin 1 morphants showed a significant increase of cilia length in KV compared with the control (Figure $7 \mathrm{G}$ and $7 \mathrm{H}$ ). To rule out the off-target effects of MO, we employed another MO targeting a different region of cofilin 1 mRNA (cofilin 1 MO-2), and observed similar phenotypes (Supplementary information, Figure S11). Furthermore, the phenotypes of Cas9/cofilin 1-gRNA-injected embryos were consistent with those of cofilin 1 morphants (Supplementary information, Figure S12). It is important to point out the similarities between the phenotypes caused by cofilin 1 depletion and NudC knockdown.

Furthermore, ectopic expression of cofilin 1 partially, but significantly, reversed the ciliary phenotypes induced by NudC depletion with either NudC MO-1 or MO-2 (Figure 7A-7H, Supplementary information, Figures S11-S13 and Movies S5-S8), suggesting that NudC may regulate ciliation and cilia-mediated developmental events by modulating cofilin 1 expression in zebrafish.

\section{Discussion}

In this study, we provide evidence that NudC plays essential roles in regulating actin cytoskeleton, ciliogenesis and zebrafish development by stabilizing cofilin 1. Actin cytoskeleton participates in many cellular processes, including muscle contraction, cell motility and cell signaling. Cofilin 1 has a pivotal role in actin dynamics by modulating actin polymerization and depolymerization through its severing activity [33-35]. Here we find that depletion of NudC causes an accumulation of bundled stress fibers and inhibits cell spreading and lamellipodia formation (Figure 1), resembling the cellular phenotypes induced by cofilin 1 knockdown [29, 30, 33]. Furthermore, our results show that NudC forms a complex with cofilin 1, colocalizes with cofilin 1 especially at the leading edge and influences the stability of cofilin 1 (Figure 4). Thus, these data suggest that NudC may act as an upstream regulator of actin cytoskeleton and dynamics by stabilizing cofilin 1 .

Recent data from our group and others have indicated that actin cytoskeleton is involved in ciliogenesis [10, $11,36,37]$. However, whether and how NudC regulates ciliogenesis remains unknown. In this report, we show that NudC depletion increases cilia length and facilitates spontaneous ciliation in mammalian cells (Figure 2). The ciliary phenotypes induced by NudC knockdown are similar to those by actin cytoskeleton disruption or cofilin 1 depletion $[10,11]$ (Figure 5). More importantly, depletion of either NudC or cofilin 1 causes similar ciliary defects in zebrafish, including body curvature, pericardial edema and defective left-right asymmetry (Figures 3 and 7 and Supplementary information, Figures S7, S10 and S11). Ectopic expression of cofilin 1 significantly reverses these phenotypes induced by NudC depletion in both cultured cells and zebrafish (Figures 6 and 7). Taken together, these findings support the hypothesis that NudC may regulate ciliogenesis via cofilin 1-mediated actin dynamics (Figure 7I).

Previous results showed that NudC is highly expressed in ciliated epithelia and localized in motile cilia [38]. Interestingly, when we examined the localization of NudC together with cilia in RPE-1 cells, we found that NudC mainly distributed in the cytoplasm, but not in cilia under our conditions (Supplementary information, Figure S14). This discrepancy suggests that NudC may have different expression patterns in different cells or tissues. The localization of NudC in motile cilia implies that NudC may have additional roles in the regulation of cilia.

As the studies from our laboratory and others indicate that NudC functions as a co-chaperone of Hsp90 to stabilize client proteins [16-18], we also investigated whether NudC regulates the stability of cofilin 1 via the Hsp90 pathway. Inhibition of Hsp90 activity by geldanamycin significantly suppressed cilia assembly (Supplementary information, Figure S15), which is consistent with the previous study [39]. Unexpectedly, geldanamycin treatment had no significant effect on the protein level of cofilin 1 (Supplementary information, Figure S16), implying that NudC may stabilize cofilin 1 via an Hsp90-independent pathway. It will be of great interest to elucidate the detailed mechanism by which NudC influences the stability of cofilin 1 .

Although depletion of either NudC or cofilin 1 in zebrafish induced the similar ciliary defects such as body 
A

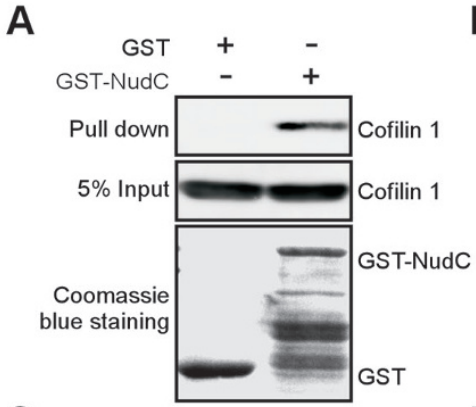

C

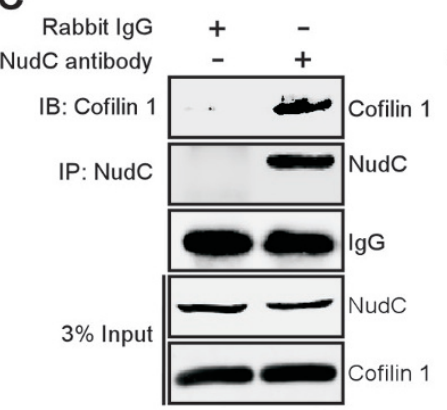

F
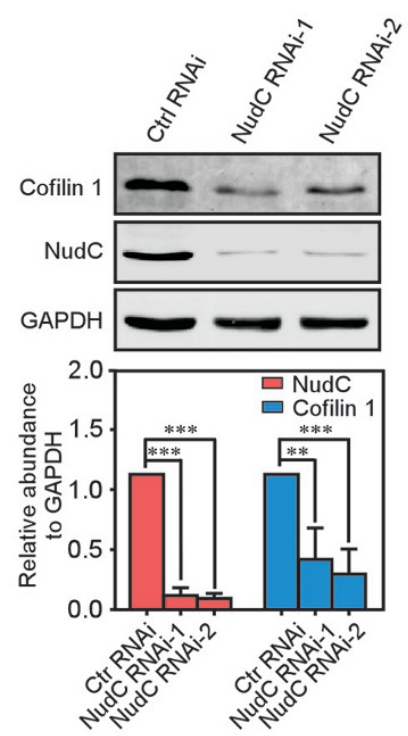

H
B

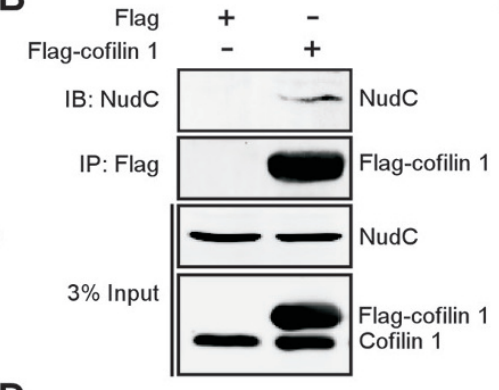

D

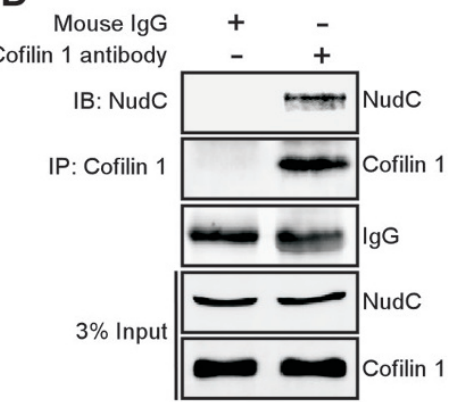

G
E
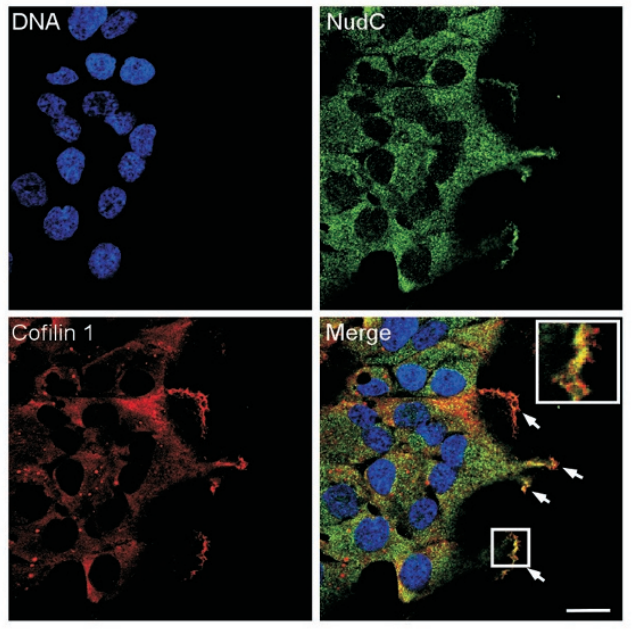

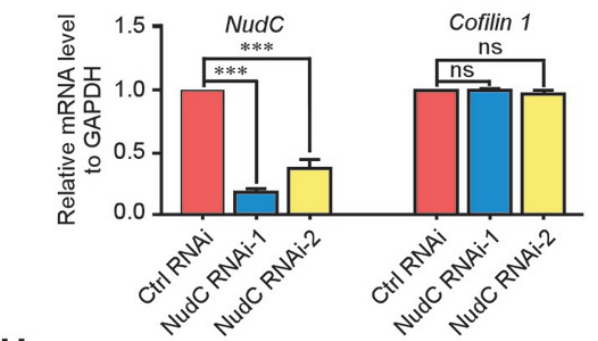

I

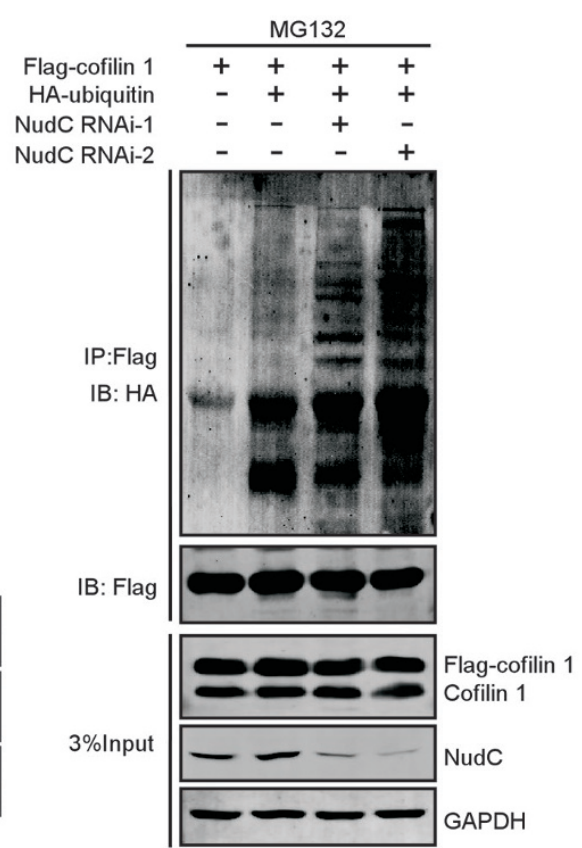

Figure 4 NudC binds to and stabilizes cofilin 1. (A) GST pull-down assay revealing the interaction between NudC and cofilin 1 in vitro. Input proteins are stained with Coomassie brilliant blue. 5\% of total input from RPE-1 cell lysates is shown. (BD) Co-immunoprecipitation confirms that NudC is associated with cofilin 1 in vivo. RPE-1 cells transfected with the indicated vectors (B) or not (C, D) are subjected to immunoprecipitation with indicated antibodies and western blotting. $3 \%$ of total input is shown. (E) Immunofluorescence revealing colocalization of NudC and cofilin 1. DNA is stained with DAPI (blue). Higher magnification of the boxed region is shown in inset. Scale bar, $20 \mu \mathrm{m}$. (F) Western analysis showing significantly decreased levels of cofilin 1 in NudC-depleted cells. Band intensities are quantified using Image J software. (G) Quantitative RT-PCR revealing that NudC RNAi has no significant effect on cofilin 1 mRNA. GAPDH is the internal control. (H) Proteasome inhibitor MG132 reverses cofilin 1 degradation induced by NudC depletion. RPE-1 cells treated with or without NudC RNAi were incubated with either $5 \mu \mathrm{M}$ MG132 or DMSO (vehicle), and subjected to western blotting. (I) NudC depletion induces the ubiquitination of cofilin 1. RPE-1 cells depleted of NudC or not were transfected with the indicated vectors and treated with 5 $\mu \mathrm{M}$ MG132. Immunoprecipitates with anti-Flag antibody from cell lysates are analyzed by immunoblotting. $3 \%$ of total input is shown Quantitative data from three independent experiments are shown as mean $\pm \mathrm{SD}$. $\mathrm{n}$, sample size. ${ }^{* *} P<0.01 ; * * * P<$ 0.001 ; ns, not significant $(P>0.05)$. 

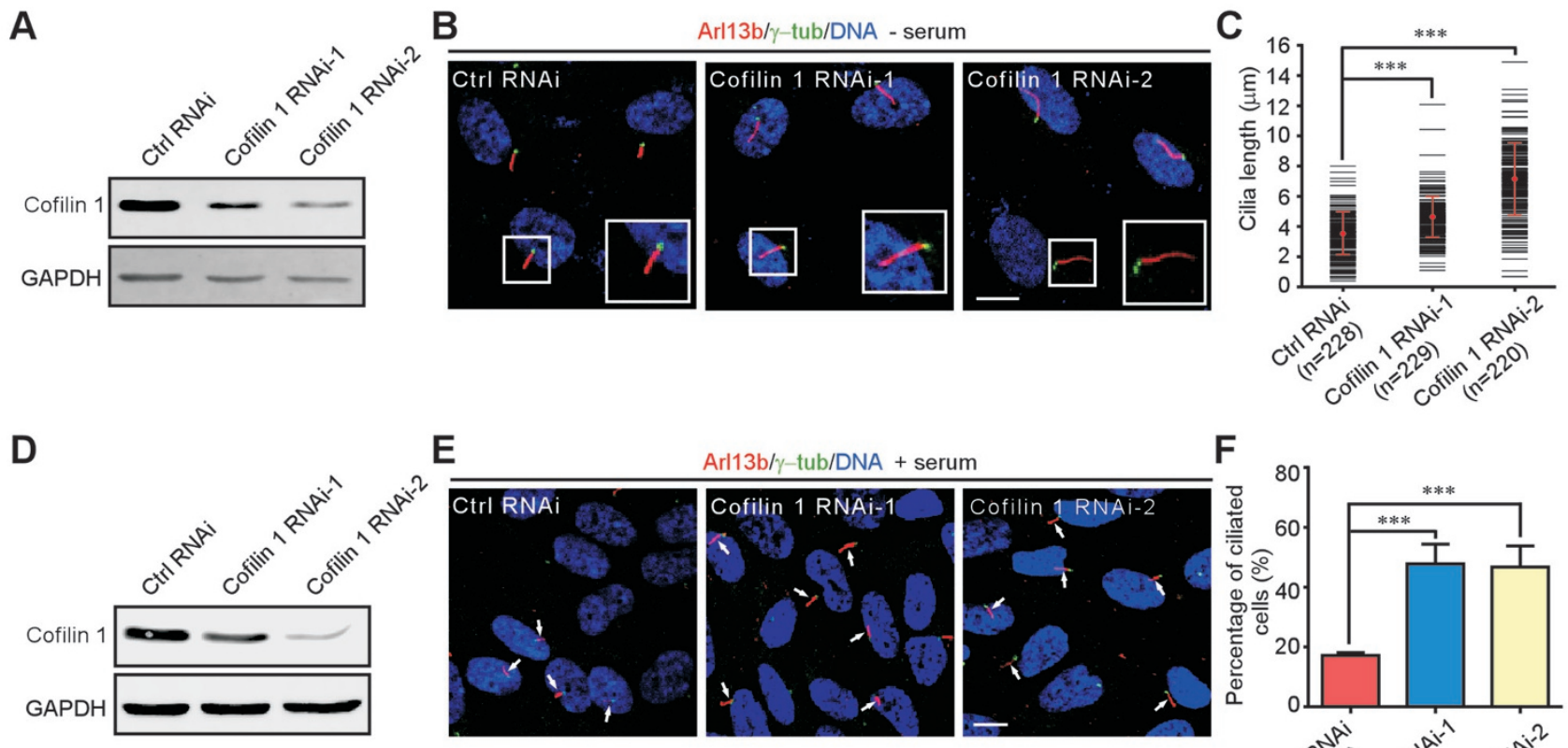

Arl13b/y-tub/DNA + serum
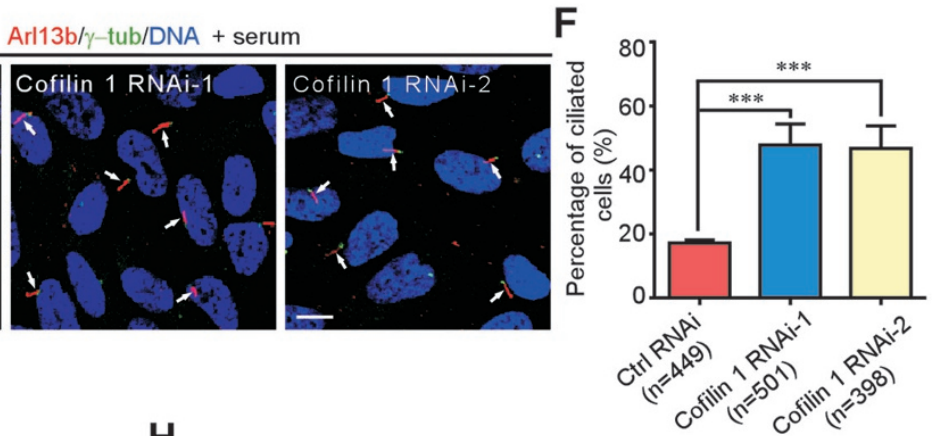

G

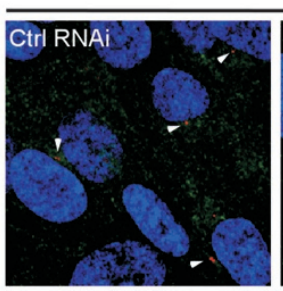

Ace-tub/Rab11a/DNA
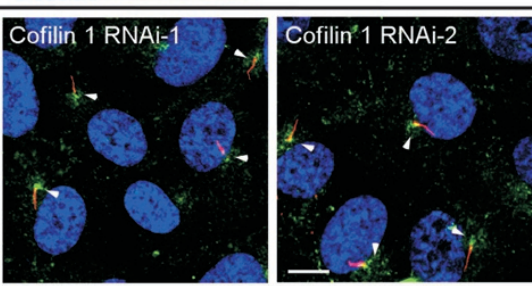

$\mathrm{H}$

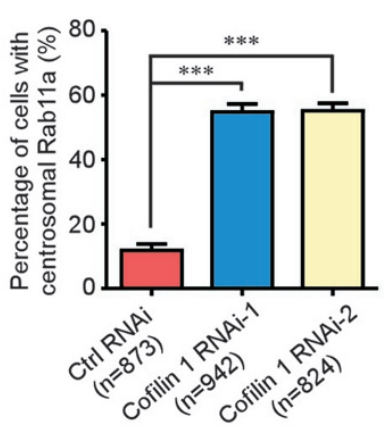

I

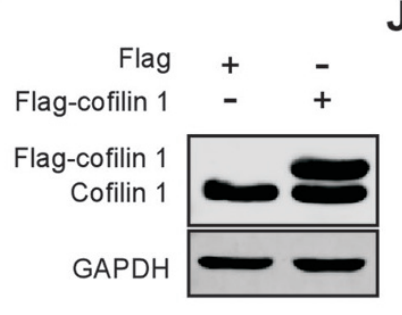

J

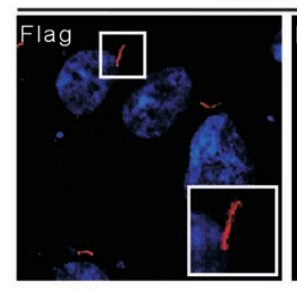

Arl13b/DNA

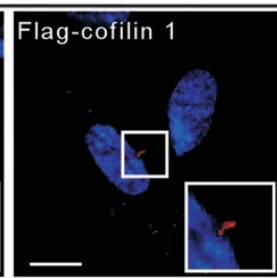

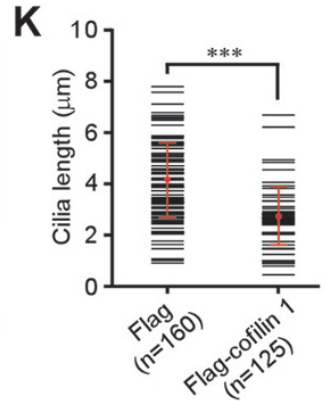

L

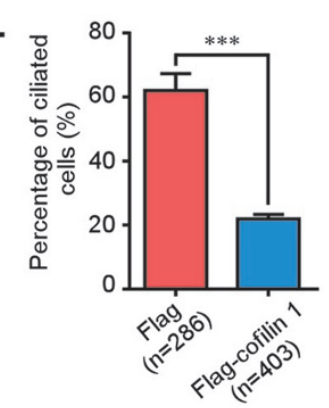

Figure 5 Cofilin 1 suppresses cilia assembly in mammalian cells. (A-C) RPE-1 cells were transfected with siRNA oligos, cofilin 1 RNAi-1 or -2, targeting different regions of cofilin 1 mRNA. Western blotting shows efficient knockdown of cofilin 1 (A). GAPDH is the internal control. Immunofluorescence with anti-Arl13b and $\gamma$-tubulin $(\gamma$-tub) antibodies reveals an increase in cilia length after cofilin 1 depletion (B). Insets are higher magnifications of the boxed areas. Cilia length (more than 70 cilia in each experiment) is scored using Image $\mathrm{J}$ software (C). (D-H) RPE-1 cells were treated with cofilin 1 RNAi. Western blotting confirms significant depletion of cofilin 1 (D). Immunostaining with antibodies reveals spontaneous ciliogenesis (arrows) in cofilin 1 knockdown cells (E). Percentage of cells with cilia in each treatment (F). Immunofluorescence reveals Rab11a-positive vesicles are frequently accumulated around the centrosome regions (arrowheads) in cofilin 1-depleted cells (G, H). More than 150 cells are scored each time. (I-L) Overexpression of cofilin 1 suppresses ciliogenesis. Western blotting (I) and immunostaining $(\mathrm{J})$ of RPE-1 cells transfected with the indicated vectors. Insets show higher magnifications of cilia. Cilia length (more than 40 cilia in each experiment) is measured using Image $\mathrm{J}$ software (K). Percentage of cells with cilia in each treatment (L). DNA is labeled with DAPI. Scale bars represent $10 \mu \mathrm{m}$. Quantitative data from at least three independent experiments are shown as mean \pm SD. $\mathrm{n}$, sample size. ${ }^{* * *} P<0.001$. 
A

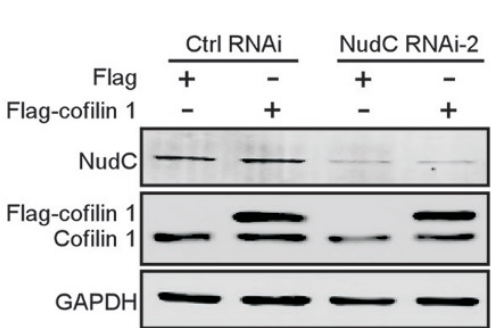

B

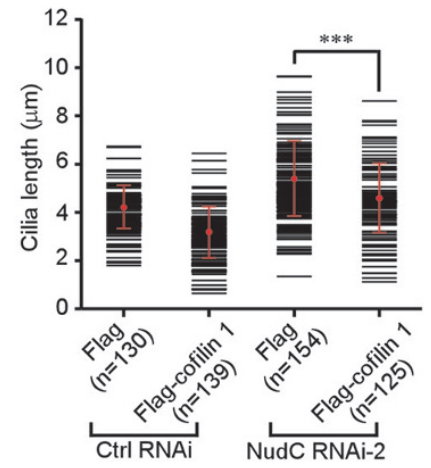

G

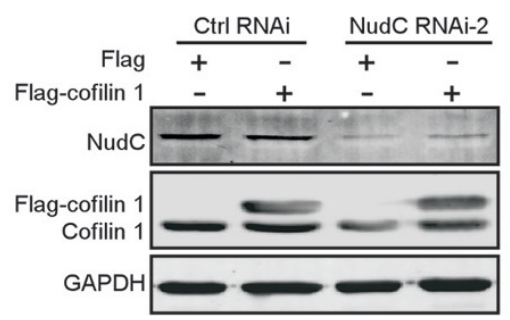

H

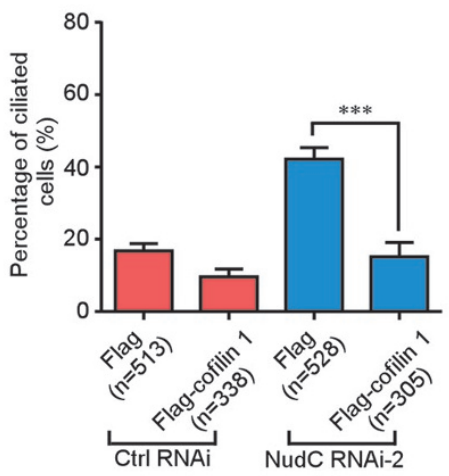

C

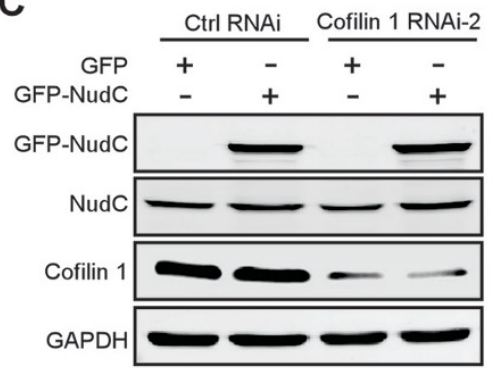

D

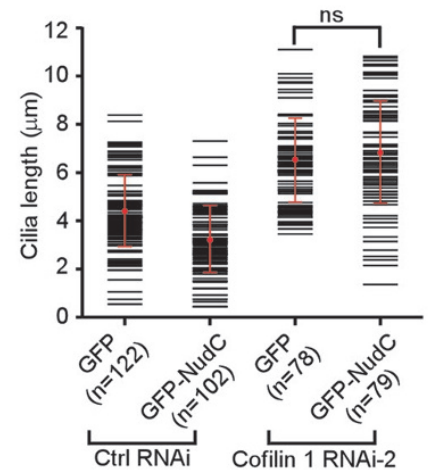

I

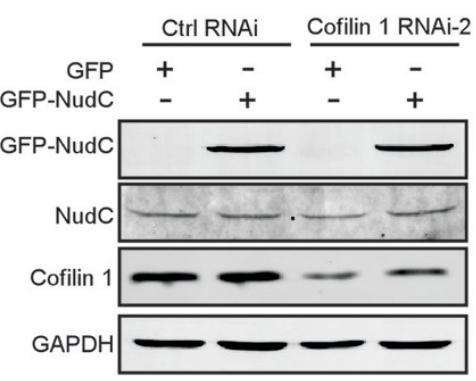

J

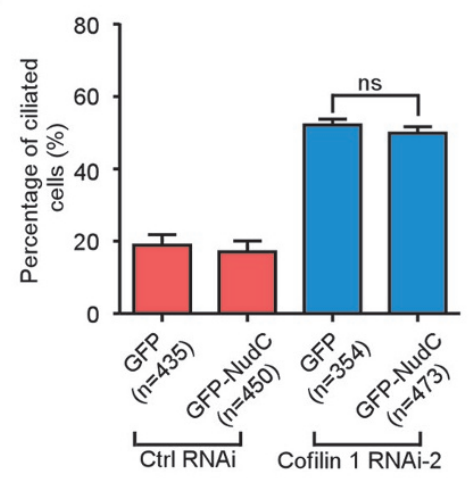

E

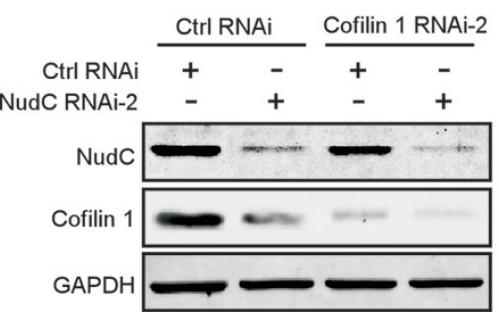

$\mathbf{F}$

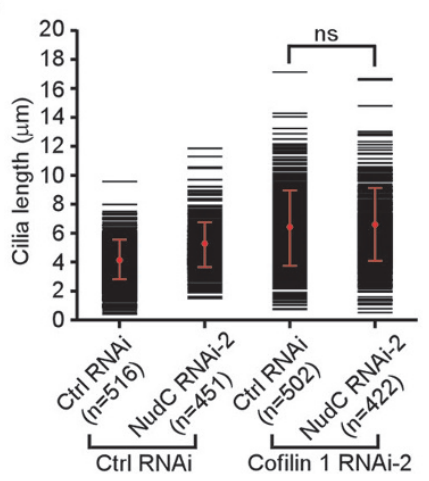

K

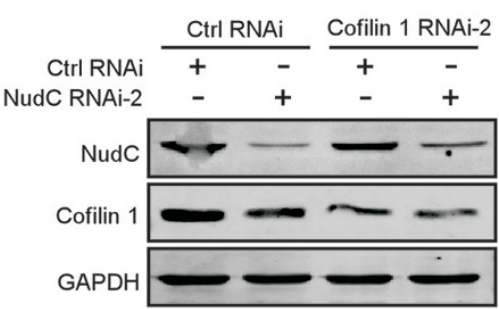

L

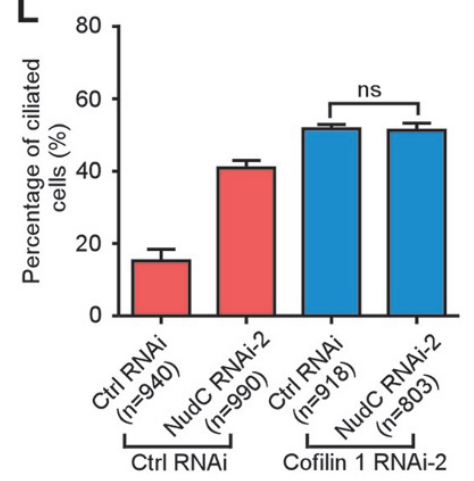

Figure 6 Cofilin 1 rescues the ciliary defects induced by NudC depletion, but not vice versa. (A-F) RPE-1 cells treated with the indicated RNAi and expression vectors and subjected to serum starvation are analyzed by western blotting and immunofluorescence. Cilia length is determined by Image $\mathrm{J}$ software. Ectopic expression of cofilin 1 reverses abnormal cilia elongation caused by NudC depletion (A, B). Exogenous expression of NudC fails to inhibit aberrant cilia elongation induced by cofilin 1 knockdown (C, D). Double depletion of NudC and cofilin 1 has no synergistic effect on cilia length (E, F). (G-L) RPE1 cells treated with the indicated RNAi and expression vectors are analyzed by immunoblotting and immunostaining. Percentages of cells with cilia in different treatments are shown. Ectopic expression of cofilin 1 inhibits spontaneous ciliogenesis caused by NudC knockdown (G, H). Exogenous expression of NudC is unable to reverse spontaneous ciliogenesis induced by cofilin 1 depletion (I, J). NudC knockdown shows no synergistic effect with cofilin 1 depletion on ciliation (K, L). Quantitative data derived from at least three independent experiments are shown as mean $\pm \mathrm{SD}$. $\mathrm{n}$, sample size. ${ }^{* * *} P<0.001$; ns, not significant $(P>0.05)$. 
curvature, pericardial edema and defective left-right asymmetry, cofilin 1 morphants did not exhibit hydrocephalus and kidney cysts that were observed in NudC morphants (Figures 3 and 7). Proteomics data from our group and others have shown that NudC may influence dozens of client proteins besides cofilin 1 [16-18], indicating that there may be cofilin 1-independent pathways involved in the role of NudC in zebrafish development. Further study is clearly needed to explore the physiological significance of NudC in vertebrates.

\section{Materials and Methods}

\section{Plasmids and oligonucleotides}

GST-NudC, GFP-NudC, Flag-cofilin 1 and Myc-NudC-R vectors were constructed as described previously [17, 40]. HA-ubiquitin and pCS107 vectors were kindly provided by Dr Ping Wang (East China Normal University) and Dr Xiao Huang (Zhejiang University), respectively. pGLV3/H1/GFP lentiviral vectors were purchased from GenePharma. To generate lentivirus expressing shRNA targeting human NudC, the following sequences were used: 5'-GAAGGGATGGCAGAGAAGC-3' (NudC RNAi-1) and 5'-AACACCTTCTTCAGCTTCCTT-3' (NudC RNAi-2). Oligos corresponding to the following sequences were synthesized by GenePharma: 5'-GGATCAAGCATGAATTGCAAGCAAA-3' for cofilin 1 RNAi-1 [41] and 5'-CATG GAAGCAGGACCAGTA-3' for cofilin 1 RNAi-2 [42]. Full-length zebrafish NudC and cofilin 1 were amplified by RT-PCR from 72 hpf zebrafish larvae and subcloned into pCS107 vector. PCR was also used to construct RNAi-resistant NudC vectors by silent mutation of five nucleic acids in the RNAi targeting region of $N u d C$ mRNA. NudC MO-1 (5'-TCTTTCGTCATCGTCACCCATTTTC-3') targeted part of the open reading frame of zebrafish $N u d C$ mRNA, the rescue construct was engineered to change the nucleotide sequence without altering the encoded protein. As NudC MO-2, cofilin $1 \mathrm{MO}-1$ and MO-2 separately target the 5'- UTR sequence of NudC and cofilin 1 mRNA, the open reading frames of these two genes were simply cloned into pCS107 vector for rescue experiments [43]. Capped and polyadenylated RNAs were synthesized with the SP6 mMessage mMachine kit (Ambion). All of these constructs were confirmed by DNA sequencing.

\section{Cell culture and transfection}

RPE-1 cells were maintained in DMEM/F12 (Corning) containing $10 \%$ serum (PAA Laboratories) at $37^{\circ} \mathrm{C}$ in $5 \% \mathrm{CO}_{2}$. HEK293T cells were cultured in DMEM (Corning) with $10 \%$ serum at $37{ }^{\circ} \mathrm{C}$ in $5 \% \mathrm{CO}_{2}$. To examine cilia length, culture medium was replaced with serum-free medium when cells were $90 \%$ confluent, then cultured for $48 \mathrm{~h}$ before use. The plasmids and synthetic oligonucleotides were transfected using Lipofectamine 2000 (Invitrogen) according to the manufacturer's instructions.

\section{Antibodies}

For IF and western blotting (WB), mouse monoclonal antibodies against acetylated tubulin (Sigma, 1:1 000 for IF), c-Myc (Santa Cruz, 1:1 000 for WB, 1:200 for IF), $\gamma$-tubulin (Sigma, 1:1 000 for IF), $\alpha$-tubulin (Sigma, 1:5 000 for WB), GAPDH (Proteintech,
1:10 000 for WB) and cofilin 1 (Proteintech, 1:1 000 for WB, 1:200 for IF) were used. Rabbit polyclonal antibodies against ARL13b (Proteintech, 1:200 for IF), CETN1 (Proteintech, 1:200 for IF) and Rab11a (Invitrogen, 1:200 for IF) were also employed. Anti-NudC antibody was generated using bacterially expressed GST-NudC as an antigen and subjected to affinity purification (Proteintech, 1:500 for WB, 1:50 for IF). Secondary antibodies for IF analyses were Alexa Fluor 488-, 568- and 647-conjugated anti-rabbit or anti-mouse IgG (Invitrogen, 1:500). Goat anti-mouse or anti-rabbit secondary antibodies (LI-COR, 1:5 000) conjugated with either Alexa Fluor 680 or IRDye 800 were used for WB.

\section{Kymography analysis}

For kymography, phase-contrast time-lapse sequences were captured using a $\times 40$ oil objective on an Olympus IX81-FV1000 confocal microscope. Images were recorded for 10-15 min at a rate of one frame per $3 \mathrm{~s}$. Kymographs were produced and analyzed by Image J software (NIH). Quantitative analysis of kymographs was performed as previously described [44].

\section{Scratch wound experiment}

Scratch wound assays were performed as described previously [45]. In brief, RPE-1 cells were plated onto fibronectin $(10 \mathrm{mg} /$ $\mathrm{ml}$ )-coated coverslips and incubated at $37{ }^{\circ} \mathrm{C}$ in $5 \% \mathrm{CO}_{2}$ to create a confluent monolayer. The monolayer was scratched using a sterile pipette tip. The cells were washed to remove the debris and then replaced with fresh medium. For lamellipodia formation assay or colocalization assay, the cells were fixed and stained after 3 $\mathrm{h}$ of scratching.

\section{Cell spreading assay}

Cell spreading assays were performed as described previously [32]. In brief, cells were resuspended in serum-free media after trypsinization and incubated at $37{ }^{\circ} \mathrm{C}$ in $5 \% \mathrm{CO}_{2}$ for $1 \mathrm{~h}$. Cells were collected and reseeded on fibronectin $(10 \mathrm{mg} / \mathrm{ml})$-coated plates. After $1 \mathrm{~h}$, cells were fixed with $4 \%$ paraformaldehyde and photographed in random fields.

\section{Flow cytometry}

For flow cytometry analysis, cells were collected, washed once with cold phosphate-buffered saline and then fixed in $70 \%$ ethanol. DNA was stained with propidium iodide (Sigma) for $30 \mathrm{~min}$ at 37 ${ }^{\circ} \mathrm{C}$. The samples were analyzed using a FC 500 MCL Flow Cytometer (Beckman Coulter).

\section{GST pull-down assay}

GST pull-down assays were performed as described previously [15]. To detect the association between NudC and cofilin 1, blots were probed with antibodies indicated in the text.

\section{Immunoprecipitation and western blotting}

Immunoprecipitation was performed as previously described $[46,47]$. In brief, cells transfected with the indicated vectors were subjected to immunoprecipitation with anti-Flag antibody-coupled beads (Sigma). Endogenous NudC and cofilin 1 were immunoprecipitated with anti-cofilin 1 and NudC antibodies, respectively. Protein samples were separated by SDS-PAGE gel, transferred to polyvinylidene fluoride membrane (Millipore), incubated with indicated antibodies and detected by an enhanced chemilumines- 
A

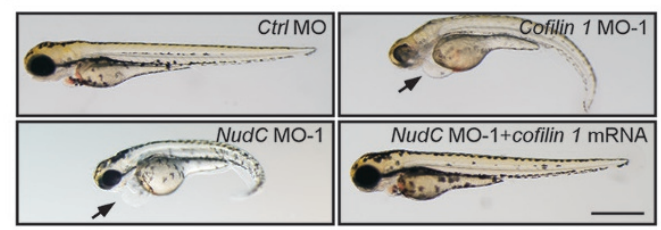

C

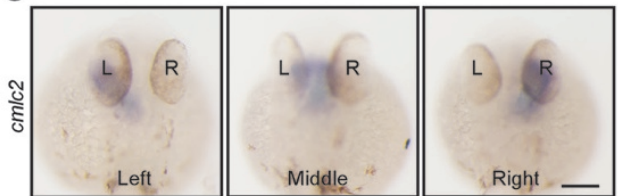

E

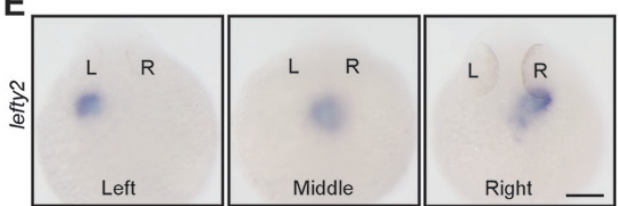

B
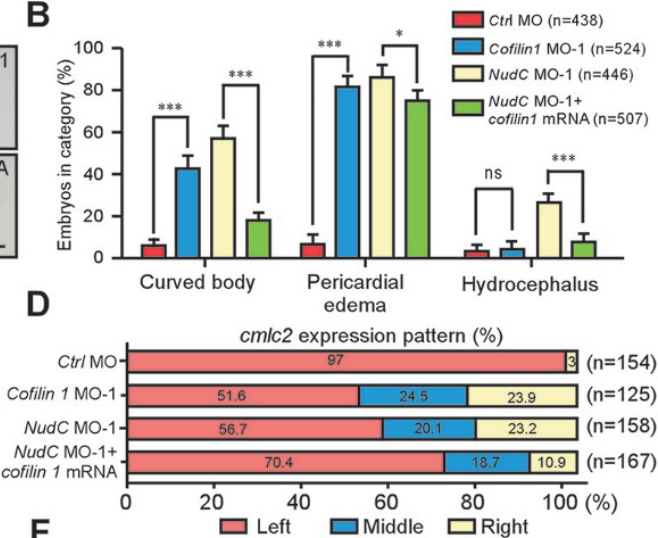

$\mathbf{F}$

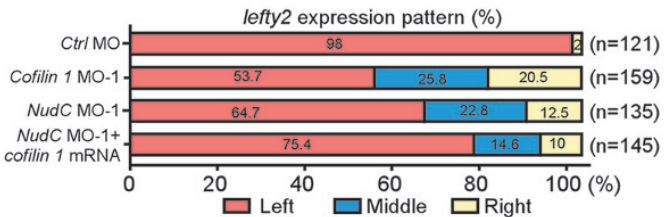

$\mathrm{H}$

G
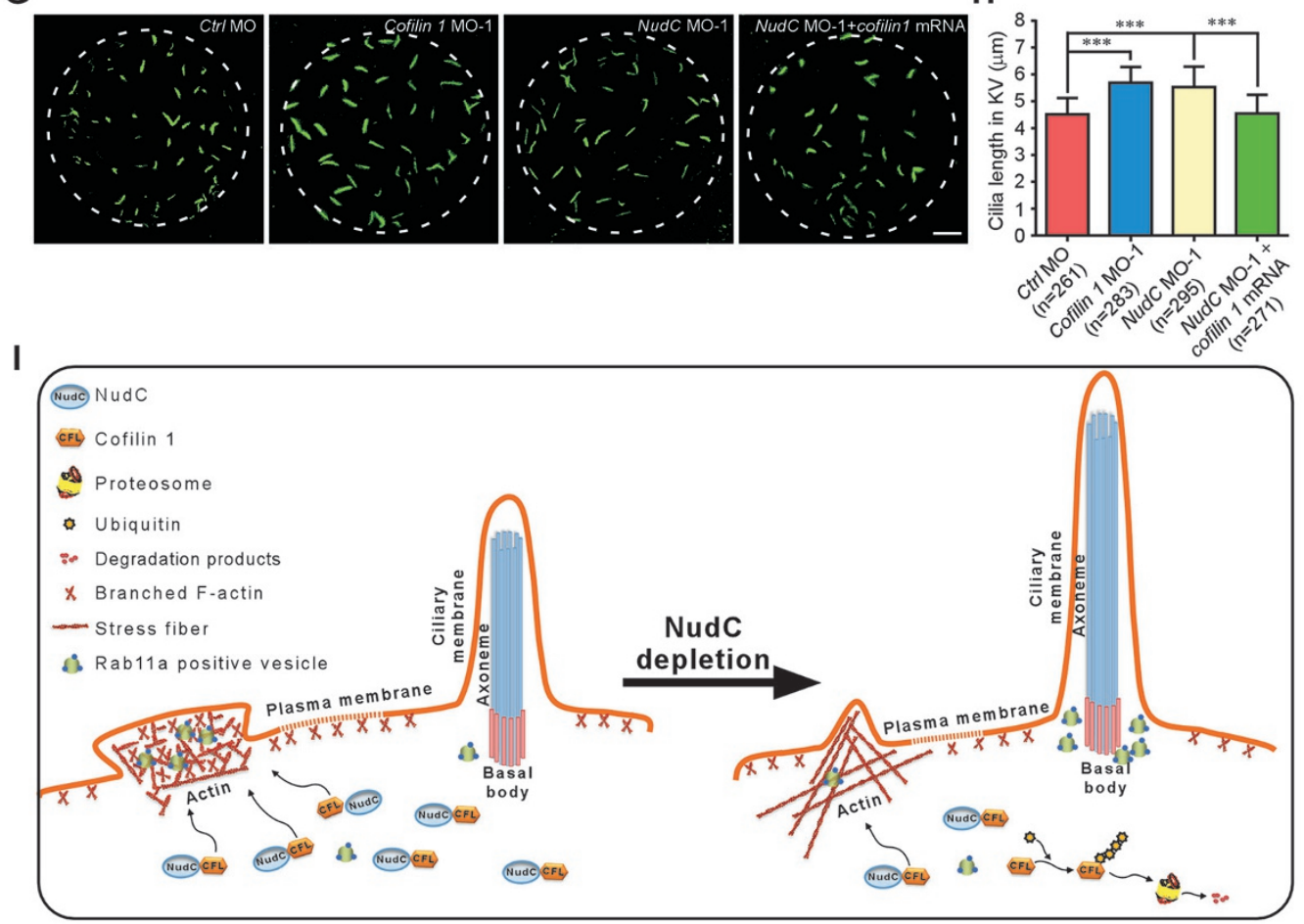

Figure 7 Cofilin 1 reverses the ciliary defects in NudC morphants. Embryos were injected with Ctrl MO (2.5 ng), NudC MO (2.5 $\mathrm{ng})$, cofilin $1 \mathrm{MO}(2.0 \mathrm{ng})$ or NudC MO (2.5 ng) plus cofilin $1 \mathrm{mRNA}(25 \mathrm{pg})$ and collected at the indicated times. (A, B) Brightfield micrographs showing body curvature and pericardial edema (arrow) in cofilin 1 morphant at $72 \mathrm{hpf}$. Ectopic expression of cofilin 1 mRNA rescues the ciliary phenotypes induced by NudC depletion. Scale bar, $500 \mu \mathrm{m}$. (C-F) Whole mount in situ hybridization with $\mathrm{cm} / \mathrm{c} 2$ at $30 \mathrm{hpf}(\mathbf{C})$ or lefty2 at $22 \mathrm{hpf}(\mathrm{E})$ reveals that the defective left-right asymmetry in NudC morphants is reversed by cofilin $1 \mathrm{mRNA}$ (dorsal view). Scale bar, $500 \mu \mathrm{m}$. Percentages of different expression patterns of $\mathrm{cm} / \mathrm{c} 2$ (D) and lefty2 (F) are also shown. (G, H) Immunofluorescence with anti-ace-tub antibody (green) at 8 ss shows that cofilin 1 mRNA rescues abnormal cilia elongation in NudC morphants. Scale bar, $10 \mu \mathrm{m}$. Cilia length in KV is measured by Image $\mathrm{J}$ software. The data are presented as mean $\pm \mathrm{SD}$ derived from at least three independent experiments. $\mathrm{n}$, sample size. $* P<0.05$; ***P $<0.001$; ns, not significant $(P>0.05)$. (I) Working model for the role of NudC in actin cytoskeleton and ciliogenesis. NudC binds to and stabilizes cofilin 1 to regulate actin dynamics and ciliogenesis. Depletion of NudC causes cofilin 1 degradation and inhibits actin dynamics possibly via the ubiquitin-proteasome pathway, resulting in accumulation of ciliogenic vesicles at basal body to facilitate cilia assembly. 
cence system (Amersham) or LI-COR Odyssey (LI-COR).

\section{Immunofluorescence staining}

Cells were grown on coverslips and fixed for 15 min with $4 \%$ paraformaldehyde. To label cilia with acetylated tubulin, cells were kept on ice for $30 \mathrm{~min}$ before fixation. Rhodamine phalloidin (1:500, Invitrogen) and 4,6-diamidino-2-phenylindole (Sigma) were used to visualize F-actin and DNA, respectively. The images were acquired using a $\times 60$ oil immersion objective (Olympus IX81-FV1000). The whole mount immunostaining of zebrafish embryos was performed as previously described [48]. The embryos mounted in $1 \%$ low melting agarose were imaged using a $60 \times$ water immersion objective (Olympus BX61W1-FV1000).

\section{Quantitative real-Time RT-PCR}

Quantitative RT-PCR analyses for NudC and cofilin 1 were performed using a Bio-Rad CFX-Touch System with PrimeScript RT reagent Kit (Takara, RR037A). All of the reactions were performed in triplicate. GAPDH served as internal control.

\section{Zebrafish maintenance and embryo production}

Wild-type zebrafish (strain AB) were maintained at $28.5^{\circ} \mathrm{C}$ using standard protocols. Zebrafish embryos were staged according to Kimmel et al. [49].

\section{Morpholinos and microinjection}

NudC MO-1 (5'-TCTTTCGTCATCGTCACCCATTTTC-3'), NudC MO-2 (5'-TTTGACGGGTGATGCTAAGAATCAC-3'), cofilin 1 MO-1 (5'-CATGGCTGTGTCTCTGTGCTAGTCG-3') and cofilin 1 MO-2 (5'-TCTGGGTTGGAATTGAATGAGCTGT-3') were designed to target the translation of $\mathrm{NudC}$ and cofilin 1 mRNA, respectively [50]. MOs were synthesized by Gene Tools and injected into zebrafish embryos at 1- to 4-cell stages at the concentration described in the text. For the rescue experiments, synthetic capped mRNAs (25 pg per embryo) were co-injected into the zebrafish embryos with MOs.

\section{In situ hybridization}

Whole mount in situ hybridization was performed as described previously [51]. In brief, zebrafish embryos and larvae were fixed with $4 \%$ paraformaldehyde at $4{ }^{\circ} \mathrm{C}$ overnight, hybridized at $68{ }^{\circ} \mathrm{C}$ with $\mathrm{cmlc} 2$ or lefty2 RNA probe labeled by DIG RNA labeling kit and then stained with BM purple (Roche).

\section{Histology}

Zebrafish larvae were fixed at $72 \mathrm{hpf}$ in $4 \%$ paraformaldehyde at room temperature for 2-3 h, dehydrated with ethanol, progressively infiltrated and embedded in paraffin. Sections $(4 \mu \mathrm{m})$ were then stained with hematoxylin and eosin.

\section{High-speed videomicroscopy}

Zebrafish larvae (48 hpf) were placed in egg water containing $40 \mathrm{mmol} / 1$ 2,3-butanedione monoxime (Sigma) to stop the heartbeat and circulation, embedded in a low melting point agarose on a glass slide, and overlaid with a drop of egg water containing 20 mmol/1 2,3-butanedione monoxime. The images were captured using a $\times 60$ oil immersion objective on an Olympus IX71 microscope equipped with a high-speed ANDOR Neo sCMOS digital video camera (Photron) at 250 frames per second for $2 \mathrm{~s}$.

\section{Statistics}

Data are representative of at least three independent experiments. Means and SDs were calculated and are shown in the graphs. The indicated $P$ values were obtained with one-way ANOVA using GraphPad Prism (GraphPad Software).

\section{Acknowledgments}

We thank Ping Wang for providing us the HA-ubiquitin expression vector; Xiao Huang for pCS107 vector. We are grateful to Yibin Kang, Lynn W Enquist and Lu Zhao for helpful suggestions; Lei Zhu, Yirong Zhang, Biao Zhu and Yijun Liu for technical assistance. We thank State Key Laboratory of Cell Biology, Institute of Biochemistry and Cell Biology, Shanghai Institutes for Biological Sciences and Chinese Academy of Sciences for instrument support. This work was supported by Ministry of Science and Technology of China (2012CB945004, 2013CB945603 and 2011CBA01001), National Natural Science Foundation of China (31125017, 31190063, 31100975, 31571446 and 31471259), Ministry of Education of China (20110101110103), Natural Scientific Foundation of Zhejiang Province, China (LY14C070001 and LY14C070002), the 111 Project (B13026), Department of Science and Technology of Zhejiang Province (2012C37089 and 2013TD13) and Zhejiang Provincial Program for the Cultivation of High-level Innovative Health talents.

\section{Author Contributions}

$\mathrm{CZ}, \mathrm{WZ}$ and YL designed the experiments. CZ and YL performed the experiments on cell biology. WZ performed zebrafish experiments. TZ conceived and directed the project. CZ, WZ, YL, YY, XZ, XY, WL and TZ analyzed data and wrote the manuscript.

\section{Competing Financial Interests}

The authors declare no competing financial interests.

\section{References}

1 Nigg EA, Raff JW. Centrioles, centrosomes, and cilia in health and disease. Cell 2009; 139:663-678.

2 Kim S, Dynlacht BD. Assembling a primary cilium. Curr Opin Cell Biol 2013; 25:506-511.

3 Panizzi JR, Becker-Heck A, Castleman VH, et al. CCDC103 mutations cause primary ciliary dyskinesia by disrupting assembly of ciliary dynein arms. Nat Genet $2012 ; 44$ :714-719.

4 Stooke-Vaughan GA, Huang P, Hammond KL, Schier AF, Whitfield TT. The role of hair cells, cilia and ciliary motility in otolith formation in the zebrafish otic vesicle. Development 2012; 139:1777-1787.

5 Zhao L, Yuan S, Cao Y, et al. Reptin/Ruvb12 is a Lrrc6/ Seahorse interactor essential for cilia motility. Proc Natl Acad Sci USA 2013; 110:12697-12702.

6 Fliegauf M, Benzing T, Omran H. When cilia go bad: cilia defects and ciliopathies. Nat Rev Mol Cell Biol 2007; 8:880-893.

7 Goetz SC, Anderson KV. The primary cilium: a signalling centre during vertebrate development. Nat Rev Genet 2010; 11:331-344.

8 Nigg EA, Cajanek L, Arquint C. The centrosome duplication cycle in health and disease. FEBS Lett 2014; 588:2366-2372.

9 Li Y, Ling K, Hu J. The emerging role of Arf/Arl small 
GTPases in cilia and ciliopathies. J Cell Biochem 2012; 113:2201-2207.

10 Kim J, Lee JE, Heynen-Genel S, et al. Functional genomic screen for modulators of ciliogenesis and cilium length. $\mathrm{Na}$ ture 2010; 464:1048-1051.

11 Cao J, Shen Y, Zhu L, et al. miR-129-3p controls cilia assembly by regulating CP110 and actin dynamics. Nat Cell Biol 2012; 14:697-706.

12 Xiang X, Fischer R. Nuclear migration and positioning in filamentous fungi. Fungal Genet Biol 2004; 41:411-419.

13 Willins DA, Xiang X, Morris NR. An alpha tubulin mutation suppresses nuclear migration mutations in Aspergillus nidulans. Genetics 1995; 141:1287-1298.

14 Aumais JP, Williams SN, Luo W, et al. Role for NudC, a dynein-associated nuclear movement protein, in mitosis and cytokinesis. J Cell Sci 2003; 116:1991-2003.

15 Zhou T, Aumais JP, Liu X, Yu-Lee LY, Erikson RL. A role for Plk1 phosphorylation of NudC in cytokinesis. Dev Cell 2003; 5:127-138.

16 Zheng M, Cierpicki T, Burdette AJ, et al. Structural features and chaperone activity of the NudC protein family. J Mol Biol 2011; 409:722-741.

17 Zhu XJ, Liu X, Jin Q, Cai Y, Yang Y, Zhou T. The L279P mutation of nuclear distribution gene $\mathrm{C}(\mathrm{NudC})$ influences its chaperone activity and lissencephaly protein 1 (LIS1) stability. J Biol Chem 2010; 285:29903-29910.

18 Taipale M, Tucker G, Peng J, et al. A quantitative chaperone interaction network reveals the architecture of cellular protein homeostasis pathways. Cell 2014; 158:434-448.

19 Spalluto C, Wilson DI, Hearn T. Evidence for reciliation of RPE1 cells in late G1 phase, and ciliary localisation of cyclin B1. Febs Open Bio 2013; 3:334-340.

20 Yan X, Zhu X. Branched F-actin as a negative regulator of cilia formation. Exp Cell Res 2013; 319:147-151.

21 Seeley ES, Nachury MV. The perennial organelle: assembly and disassembly of the primary cilium. J Cell Sci 2010; 123:511-518.

22 Ishikawa H, Marshall WF. Ciliogenesis: building the cell's antenna. Nat Rev Mol Cell Biol 2011; 12:222-234.

23 Kishimoto N, Cao Y, Park A, Sun Z. Cystic kidney gene seahorse regulates cilia-mediated processes and Wnt pathways. Dev Cell 2008; 14:954-961.

24 Juric-Sekhar G, Adkins J, Doherty D, Hevner RF. Joubert syndrome: brain and spinal cord malformations in genotyped cases and implications for neurodevelopmental functions of primary cilia. Acta Neuropathol 2012; 123:695-709.

25 Essner JJ, Amack JD, Nyholm MK, Harris EB, Yost HJ. Kupffer's vesicle is a ciliated organ of asymmetry in the zebrafish embryo that initiates left-right development of the brain, heart and gut. Development 2005; 132:1247-1260.

26 Burdine RD, Caspary T. Left-right asymmetry: lessons from Cancun. Development 2013; 140:4465-4470.

27 Chang NN, Sun CH, Gao L, et al. Genome editing with RNA-guided Cas9 nuclease in Zebrafish embryos. Cell Res 2013; 23:465-472.

28 Chan AY, Bailly M, Zebda N, Segall JE, Condeelis JS. Role of cofilin in epidermal growth factor-stimulated actin polymerization and lamellipod protrusion. J Cell Biol 2000; 148:531542.
29 Hotulainen P, Paunola E, Vartiainen MK, Lappalainen P. Actin-depolymerizing factor and cofilin-1 play overlapping roles in promoting rapid $\mathrm{F}$-actin depolymerization in mammalian nonmuscle cells. Mol Biol Cell 2005; 16:649-664.

30 Wiggan O, Shaw AE, DeLuca JG, Bamburg JR. ADF/cofilin regulates actomyosin assembly through competitive inhibition of myosin II binding to F-actin. Dev Cell 2012; 22:530-543.

31 Goldberg AL. Protein degradation and protection against misfolded or damaged proteins. Nature 2003; 426:895-899.

32 Yoo Y, Ho HJ, Wang C, Guan JL. Tyrosine phosphorylation of cofilin at Y68 by v-Src leads to its degradation through ubiquitin-proteasome pathway. Oncogene 2010; 29:263-272.

33 Wang W, Eddy R, Condeelis J. The cofilin pathway in breast cancer invasion and metastasis. Nat Rev Cancer 2007; 7:429440.

34 Bravo-Cordero JJ, Magalhaes MA, Eddy RJ, Hodgson L, Condeelis J. Functions of cofilin in cell locomotion and invasion. Nat Rev Mol Cell Biol 2013; 14:405-415.

35 Bernstein BW, Bamburg JR. ADF/cofilin: a functional node in cell biology. Trends Cell Biol 2010; 20:187-195.

36 Chhabra ES, Higgs HN. The many faces of actin: matching assembly factors with cellular structures. Nat Cell Biol 2007; 9:1110-1121.

37 Goley ED, Welch MD. The ARP2/3 complex: an actin nucleator comes of age. Nat Rev Mol Cell Biol 2006; 7:713-726.

38 Gocke CD, Osmani SA, Miller BA. The human homologue of the Aspergillus nuclear migration gene nudC is preferentially expressed in dividing cells and ciliated epithelia. Histochem Cell Biol 2000; 114:293-301.

39 Prodromou NV, Thompson CL, Osborn DP, et al. Heat shock induces rapid resorption of primary cilia. J Cell Sci 2012; 125:4297-4305.

40 Shen M, Cai Y, Yang Y, Yan X, Liu X, Zhou T. Centrosomal protein FOR20 is essential for S-phase progression by recruiting Plk1 to centrosomes. Cell Res 2013; 23:1284-1295.

41 von Blume J, Duran JM, Forlanelli E, et al. Actin remodeling by $\mathrm{ADF} /$ cofilin is required for cargo sorting at the trans-Golgi network. J Cell Biol 2009; 187:1055-1069.

42 Klamt F, Zdanov S, Levine RL, et al. Oxidant-induced apoptosis is mediated by oxidation of the actin-regulatory protein cofilin. Nat Cell Biol 2009; 11:1241-1246.

43 Bill BR, Petzold AM, Clark KJ, Schimmenti LA, Ekker SC. A primer for morpholino use in zebrafish. Zebrafish 2009; 6:6977.

44 Bear JE, Svitkina TM, Krause M, et al. Antagonism between Ena/VASP proteins and actin filament capping regulates fibroblast motility. Cell 2002; 109:509-521.

45 Liang CC, Park AY, Guan JL. In vitro scratch assay: a convenient and inexpensive method for analysis of cell migration in vitro. Nat Protoc 2007; 2:329-333.

46 Yang Y, Yan X, Cai Y, Lu Y, Si J, Zhou T. NudC-like protein 2 regulates the LIS1/dynein pathway by stabilizing LIS1 with Hsp90. Proc Natl Acad Sci USA 2010; 107:3499-3504.

47 Yang YF, Ran J, Liu M, et al. CYLD mediates ciliogenesis in multiple organs by deubiquitinating Cep70 and inactivating HDAC6. Cell Res 2014; 24:1342-1353.

48 Wloga D, Webster DM, Rogowski K, et al. TTLL3 is a tubulin glycine ligase that regulates the assembly of cilia. Dev Cell 2009; 16:867-876. 
49 Kimmel CB, Ballard WW, Kimmel SR, Ullmann B, Schilling TF. Stages of embryonic development of the zebrafish. Dev Dyn 1995; 203:253-310.

50 Lin CW, Yen ST, Chang HT, et al. Loss of cofilin 1 disturbs actin dynamics, adhesion between enveloping and deep cell layers and cell movements during gastrulation in zebrafish. PLoS One 2010; 5:e15331.

51 Oxtoby E, Jowett T. Cloning of the zebrafish krox-20 gene $(\mathrm{krx}-20)$ and its expression during hindbrain development. Nucleic Acids Res 1993; 21:1087-1095.

(Supplementary information is linked to the online version of the paper on the Cell Research website.) 